\title{
Therapeutic endoscopic strategies in early esophageal cancer and dysplastic Barrett's epithelium
}

\author{
Teresa Fritz $(\mathbb{D}) \cdot$ Rainer Schöfl · Friedrich Wewalka · Alexander Ziachehabi
}

Received: 31 July 2018 / Accepted: 7 November 2018 / Published online: 5 December 2018

(C) The Author(s) 2018

\begin{abstract}
Summary Endoscopic therapy is the gold standard for curative treatment of early esophageal adenocarcinoma (EAC) including dysplastic Barrett's epithelium (BE) and very early squamous cell carcinoma (SCC) because it is superior to surgery in regard to morbidity, mortality and cost effectiveness while yielding excellent results and low complication rates.

Tumor detection at an early stage is often challenging and a multimodal approach using high resolution white light endoscopy, virtual chromoendoscopy (e.g. narrow band imaging, NBI) and endoscopic ultrasonography (EUS) is recommended. Importantly, histological diagnosis and EUS guided tumor staging should be performed before endoscopic tumor resection, although EUS validity has its limitations in terms of superficial mucosal and submucosal tumor invasion.

In early esophageal adenocarcinoma, endoscopic mucosal resection (EMR) is considered the first line therapy and endoscopic submucosal dissection (ESD) is recommended only in special cases. In contrast, in very early squamous cell carcinoma, ESD is superior to EMR. This is mainly caused by a lower risk for lymphatic metastases in adenocarcinoma compared to squamous cell carinoma. If endoscopic resection is not curative or not feasible, surgery is the treatment of choice - assuming the patient's comorbidities and performance status are no exclusion criteria.
\end{abstract}

Keywords ESD - EMR · EUS · Esophageal cancer

T. Fritz $(\bowtie) \cdot$ R. Schöfl · F. Wewalka · A. Ziachehabi

4. Interne Abteilung, Ordensklinikum Linz/Elisabethinen,

Fadingerstraße 1, 4020 Linz, Austria

teresa.fritz@ordensklinikum.at

\section{Introduction}

Since esophageal tumor surgery is associated with a high adverse events rate [1], endoscopic local tumor therapy as a minimally invasive therapy option has continuously attracted increasing attention during the past decade. Today, endoscopic therapy is recognized as the therapy of choice for very early squamous cell carcinoma (SCC) and early esophageal adenocarcinoma (EAC) by gastroenterology societies but it is restricted to expert centers because of high technical skill demands.

Endoscopic tumor therapy originates from piecemeal endoscopic mucosal resection (EMR) and developed to endoscopic submucosal dissection (ESD).

Injection-assisted EMR was introduced for flexible colonoscopy in 1973 and allows for the removal of suspicious mucosal lesions-smaller ones in one piece, bigger ones in multiple pieces-throughout the gastrointestinal tract [2]. The problem hereby is-due to the multiple pieces-the limitation of histological staging and a higher potential for recurrence of neoplastic lesions since en bloc EMR is practicable only for lesions of $\leq 15-20 \mathrm{~mm}$ in diameter.

ESD was developed in Japan and is a technique that allows for en bloc resection of visible mucosal and superficial submucosal lesions irrespective of their size $[3,4]$. Originally, it was used to dissect neoplastic lesions in the stomach, but since this procedure was so successful regarding effectiveness and safety, it was finally extended for use in the esophagus and colorectum.

Compared with EMR, ESD is more time consuming and associated with higher complication rates [5, 6], but en bloc resection of large lesions infiltrating the superficial submucosal layer is feasible and it facilitates appropriate histological evaluation. Moreover, 
ESD is superior to surgery in terms of morbidity, mortality [7], and cost efficacy [8].

\section{Staging}

Before endoscopic tumor therapy is initiated, the neoplasia should be histologically verified. Endoscopic ultrasound (EUS)-guided tumor staging plays a leading role to exclude deep tumor infiltration of the esophageal wall, but is limited in distinguishing superficial from deep submucosal tumor infiltration, implying a relevant risk for EUS-guided tumor underor overstaging [9].

In a meta-analysis of 49 studies $(n=2558)$, the pooled sensitivity and specificity of EUS for detection of $\mathrm{T} 1$ tumors $(81.6 \%$ and $99.4 \%$, respectively) and for $\mathrm{T} 2$ tumors (81.4\% and $96.3 \%$, respectively) revealed the inferiority of EUS in early tumor stages compared with more advanced tumor stages, since $\mathrm{T} 3$ and T4 tumors were revealed to have the highest pooled sensitivity and specificity rates $(91.4 \%$ and $94.4 \%$ for T3 and $92.4 \%$ and $97.4 \%$ for T4 tumors, respectively). Moreover, it could be demonstrated that the use of fine needle aspiration (FNA) could improve lymph node staging (sensitivity $84.7 \%$ vs. $96.7 \%$ and specificity $84.6 \%$ vs. $95.5 \%$, respectively; [10]).

On the other hand, Thomas et al. and others [11, 12] showed that flat, non-nodular lesions in Barrett's epithelium were limited exclusively to the mucosal layer, so that EUS had no benefit and should be reconsidered in this special selected cohort [9].

Therefore, it is crucial for endoscopists to learn how to distinguish lesions infiltrating the superficial submucosa from intraepithelial tumors macroscopically. This is done by a combination of high-resolution white light endoscopy and virtual chromoendoscopy with magnification (e.g., NBI).

Submucosal invasion is often associated with significant morphological changes as these lesions mainly appear elevated and/or excavated with a destruction of their mucosal structure (e.g., ulcerations) [13]. By contrast, intramucosal tumors generally appear flat without a significant effect on the mucosal surface. Importantly, these macroscopic criteria alone are insufficient to distinguish between mucosal and submucosal invasion. Additionally, magnification endoscopy together with virtual chromoendoscopy (e.g., NBI, narrow-band imaging) should therefore be performed.

Narrow-band imaging yields a high contrast image for the evaluation of squamous cell tissue for irregular intrapapillary capillary loops (IPCLs) [14].

Intrapapillary capillary loops are microvessels in the squamous cell mucosa and they are an indicator of tissue atypia if special vessel patterns occur (dilatation, meandering, caliber change, and non-uniformity in the appearance of each IPCL). These vessel patterns mark a destruction of the IPCL structure and help to distinguish between tumorous and non-tumorous/ inflamed tissue as well as between intramucosal and submucosal tumor invasion [15, 16].

The evaluation of a suspicious lesion using IPCL classification is therefore an indispensable tool in making a decision on which therapeutic strategy should be followed in early esophageal squamous cell carcinoma.

In Barrett's epithelium with dysplastic or tumorsuspicious visible lesions, endoscopists should also perform magnifying NBI-guided endoscopy to evaluate the mucosal microsurface and microvascular pit pattern.

In general, non-dysplastic Barrett's mucosa presents with a regular surface pit pattern including rounded, circular, or oval crypts. If an irregular or disrupted mucosal pit pattern occurs, dysplasia or invasive cancer must be suspected [17].

Additionally, the microvascular pattern must be evaluated since abnormal blood vessels (irregular, dilated, corkscrew type vessels) are a further sign of the presence of dysplasia or cancer. To date, there are several classifications for pathologic microvascular and microsurface patterns available [18, 19], but to date, there is no consensus on how to clearly distinguish mucosal from submucosal tumor infiltration in Barrett's adenocarcinoma.

This underlines the importance of the EMR procedure as a powerful staging mechanism, and sceptics of EUS argue that early Barrett's neoplasia can be staged accurately by histological assessment of EMR specimens [20] and that surgery can be added if no vertical $\mathrm{R} 0$ resection is achieved.

\section{Endoscopic strategies for dysplastic Barrett's ep- ithelium and EAC}

In the West, the incidence of esophageal adenocarcinoma (EAC) has dramatically increased during the past decades [21]. Adenocarcinoma arises from metaplastic Barrett's epithelium in the distal esophagus and is mainly a result of gastroesophageal reflux disease (GERD).

Over time, Barrett's epithelium can transform via low-grade dysplastic mucosa and high-grade dysplasia to invasive cancer, but the estimated risk of cancer progression from nondysplastic Barrett's epithelium is low $(0.3 \%$ per year) [22]. Therefore, screening endoscopy only in patients with longstanding GERD and other risk factors (age $\geq 50$ years, obesity, smoking, male sex, fist-degree relative with Barrett's epithelium/adenocarcinoma) is recommended. Screening should be repeated every 3-5 years if Barrett's metaplasia is diagnosed to detect potential malignant epithelial transformation at an early tumor stage [23]. Unfortunately, however, only 5-7\% of patients with EAC have a prior diagnosis of Barrett's epithelium [24], which highlights the limitations of this screening program, since a large proportion of patients with Barrett 
mucosa are asymptomatic and therefore remain undetected [25].

When a visible lesion suggestive of dysplasia or early invasive cancer is detected in Barrett's epithelium, it should be endoscopically removed by EMR if deep tumor invasion or lymphatic metastasis was excluded by EUS. Here, ESD is recommended only for special indications (see below) [26].

The diagnosis of any grade of dysplasia in random biopsies from Barrett's epithelium should be confirmed by an expert gastrointestinal (GI) pathologist and it is important to rule out macroscopic signs of inflammation in the Barrett's mucosa before biopsies are taken.

When high-grade intraepithelial neoplasia (HGIN) is diagnosed and confirmed by the GI expert, radiofrequency ablation (RFA) is recommended [23].

Histologic confirmation by an expert is especially crucial for patients with random low-grade intraepithelial neoplasia (LGIN), because it was demonstrated that the majority $(73 \%)$ of community-diagnosed low-grade dysplasia was downstaged by experts. On the other hand, patients with expertconfirmed low-grade dysplasia had a relevant risk for malignant disease progression ( $9.1 \%$ per patient year) [27].

Radiofrequency ablation in low-grade intraepithelial neoplasia is only recommended if it is confirmed twice within 6 months under ongoing proton pump inhibitor therapy [23]. Radiofrequency ablation sessions should be performed until Barrett's epithelium is completely eradicated.

\section{Endoscopic mucosal resection in early EAC}

To date, EMR is the gold standard for removing visible lesions in Barrett's mucosa and it is considered curative when the vertical resection margins are tumor free, when the tumor is well differentiated, and lymphovascular/vascular tumor infiltration can be histologically excluded [26]. It is usually performed as a cap-assisted (cEMR) procedure-this involves submucosal saline injection, sucking of the lifted lesion into the cap, and dissection by a cap-placed snare [28]. Alternatively, multiband mucosectomy (MBM) can be performed, using a multiband ligator system to fix the neoplastic mucosal area by deploying a rubber band around it and finally removing it underneath the rubber band by snare [29]. Both EMR techniques show similar resection depths [30], specimen diameters, and complication rates [31], while MBM was shown to be faster and less expensive [32].

\section{The role of radiofrequency ablation}

There is a critical difference in the treatment of dysplastic/neoplastic Barrett's epithelium between Eastern and Western countries, since RFA is not available in the East. The combination of EMR/ESD and RFA as a multimodal approach is the standard therapy regimen for dysplastic and neoplastic BE in the West in order to prevent metachronous neoplasia [33]. In the East, however, suspicious mucosal lesions are endoscopically removed, often without removing residual BE. In general, endoscopic resection is the only feasible therapy option in many Asian countries including Japan.

Radiofrequency ablation involves the direct application of heat to Barrett's mucosa, whether by using a special balloon device for circumferential treatment (360 ${ }^{\circ}$ ablation), or by a probe attached to the scope or a through-the-scope probe for focal treatment.

Since histological assessment after RFA treatment is invalid, all visible lesions must be resected before RFA application. Several studies demonstrated a benefit from RFA treatment vs. surveillance of Barrett's epithelium with low-grade dysplasia, resulting in a significant decrease of disease progression to highgrade dysplasia/invasive adenocarcinoma [34-36]. Long-term follow-up revealed excellent results when Barrett's epithelium was completely eradicated: After 3 years, $91 \%$ of patients presented without Barrett's mucosa and $98 \%$ of patients presented without dysplasia, while strictures occurred in $7.3 \%$ [37].

\section{Endoscopic submucosal dissection vs. endoscopic mucosal resection in EAC}

Endoscopic submucosal dissection is performed with special "knives" through the channel of the endoscope, developed by Japanese experts. After applying heat as marking dots around the neoplastic area, saline injection to the submucosa followed by a submucosal cut allows for en bloc dissection of the desired area within the submucosal layer.

Notably, ESD in early adenocarcinoma is limited to special indications. This is primarily based on the finding that predictable piecemeal mucosectomy is a high-risk factor for neoplastic recurrence rates (relative risk: 2.44 [95\% CI: 1.13-4.89], $p=0.02$; [38]).

Therefore, ESD should be considered for tumors of $\geq 15 \mathrm{~mm}$ in diameter. Moreover, ESD is recommended when poor tumor lifting is expected (e.g., if the area to be dissected contains fibrotic tissue). Finally, if superficial submucosal tumor invasion is assumed via white light and virtual chromoendoscopy (e.g., elevated, depressed, or ulcerated lesions together with a pathologic microvessel pattern; [13]) and deep tumor infiltration or lymphatic metastasis is excluded by EUS, ESD is preferred to EMR. This is because it provides a minimally invasive, curative treatment [26] if the following four criteria are met: (1) submucosal layer invasion is $\leq 500 \mu \mathrm{m}$, (2) poor tumor differentiation is excluded, (3) lymphatic/venous vessel infiltration is missing, and (4) tumor size $\leq 30 \mathrm{~mm}$. Notably, the risk of lymph node metastasis for Barrett's adenocarcinoma is significantly increased with the depth 
of tumor infiltration ( $0 \%$ in HGIN/mucosal infiltration vs. $12 \%$ in submucosal infiltration, $p=0.045$ ) [39].

Nevertheless, the absence of the aforementioned criteria is associated with a low lymphatic metastasis potential and therefore ESD can be considered curative even if the superficial submucosal layer (sm1 $\leq 500 \mu \mathrm{m}$ ) is involved [40-42].

One randomized controlled study in 2017 focused on the differences in outcome and adverse events of EMR compared with ESD: Terheggen et al. demonstrated no difference in complete remission from neoplasia at the 3-month follow-up (ESD 93.8\% vs. EMR $94.1 \%, p=1.0$ ) while ESD was associated with a higher rate of adverse events [43].

Notably, ESD yields higher en bloc resection rates (OR: 13.9, 95\% CI: 10.12-18.99; $p=0.001$ ) and higher curative resection rates (OR: $3.53,95 \% \mathrm{CI}$ : 2.57-4.84; $p=0.000)$ compared with EMR throughout the GI tract but is more time consuming and associated with higher rates of adverse events [44]. However, this advantage does not seem to have any clinical impact on the need for elective surgery or the rates of complete remission from neoplasia [43]. This explains why EMR in combination with RFA is the preferred technique to remove early EAC endoscopically.

However, both in EMR and ESD, additional surgery is recommended-depending on the patient's comorbidities and performance status-when the vertical specimen margins are infiltrated by tumor.

\section{Endoscopic strategies for very early esophageal squamous cell carcinoma}

Detection of squamous cell carcinoma in the esophagus at a very early stage is challenging since these lesions often appear subtle and are usually flat. The highest tumor detection rates can be achieved using the combination of high-resolution white light endoscopy and virtual chromoendoscopy with NBI. With regard to the detection rate of high-grade dysplasia and squamous cell carcinoma, NBI is equal to Lugol staining, but NBI has a higher specificity (82\% vs. $37 \%$ ), while sensitivity is similar [45]. Additionally, Inoue et al. demonstrated that the microvascular pattern of the IPCL can be used to predict the degree of malignancy and invasiveness of a lesion [46].

\section{ESD in early squamous cell carcinoma}

In contrast to EMR, ESD is the gold standard for removing very early esophageal squamous cell carcinoma.

The superiority of ESD to EMR in this tumor entity is based on higher en bloc resection rates [44], higher R0 resection rates $(97.4 \%$ vs. $78.3 \%, p=0.0002)$, and lower tumor recurrence rates $(0.9 \%$ vs. $9.8 \%$, $p=0.0065$; [47]). Furthermore, there is a substantial risk for lymph node metastasis in SCC, increasing to $8-18 \%$ when lamina muscularis mucosae $(\mathrm{m} 3)$ is in- filtrated, while deeper invasion bears a lymph node risk of up to $11-53 \%$ for sm 1 and $30-54 \%$ for sm 2 tumors [48-50]. This explains why ESD is considered curative only for intraepithelial (m1) and lamina propria infiltrating (m2) tumors since there is low lymphometastatic potential. Submucosal tumor invasion in squamous cell carcinoma $\leq 200 \mu \mathrm{m}$ ( $\mathrm{sml}$ ) can be tolerated if poor tumor differentiation, lymphatic or venous vessel invasion, and tumor infiltration of the vertical dissection margins can be excluded. Otherwise, patients must be evaluated for tumor surgery.

\section{Safety and management of complications in en-} doscopic tumor resection in the esophagus

Compared with esophageal resection, EMR and ESD both are considered a safe procedure associated with low complication rates. The most frequently occurring complications are bleeding, perforations, and esophageal strictures. Usually, bleeding and small perforations can be successfully treated endoscopically.

A meta-analysis of 11 studies, where 501 ESDs of early esophageal adenocarcinoma between 2005 and 2016 were analyzed, revealed a pooled perforation rate of $1.5 \%(0.4-3 \%)$, a pooled bleeding rate of $1.7 \%(95 \%$ CI: $0.6-3.4 \%$ ), and the pooled risk of stricture development was $11.6 \%$ (95\% CI: 0.9-29.6\%) while the incidence of neoplastic recurrence after curative resection was $0.17 \%$ (95\% CI: $0-0.3 \%$ ) at a mean follow-up of 22.9 months (95\% CI: 17.5-28.3) [51].

Strictures often represent a long-term issue with recurrent need for balloon dilatation treatment. Stricture development depends primarily on the resection size. A resection size including more than $75 \%$ of esophageal circumference accompanied by a tumor infiltration deeper than the lamina propria layer $(>\mathrm{m} 2)$ is significantly associated with stricture development [52]. Currently, systemic steroid therapy and local steroid injections as stricture prophylaxis after ESD in patients with relevant tumor resection size are under debate in multiple studies. To date, no guideline-based recommendation for steroid therapy has been published. Nevertheless, some studies pointed out that postinterventional systemic steroid treatment beneficially influences stricture development, suggesting that this could at least be an attempt of treatment [53-55].

Recently, another study revealed that $25 \%$ of patients who underwent ESD with circumferential resection rates of $>75 \%$ showed esophageal dysmotility in high-resolution manometry studies and $69 \%$ of these patients also presented with clinically verifiable dysmotility symptoms although no stricture could be detected endoscopically [56]. Therefore, dysmotility must also be considered as a potential adverse event after endoscopic tumor resection. 


\section{Conclusion and future perspectives}

Both in Barrett's and squamous cell early esophageal cancer, endoscopic resection is considered the therapy of choice if deep tumor infiltration, lymphatic or venous vessel infiltration, or poor tumor differentiation can be excluded [26]. As a minimally invasive tumor therapy, endoscopic resection is more cost effective [8] and associated with lower morbidity and mortality rates [7].

Stricture development after the resection of large esophageal tumors with substantial circumferential involvement is still a problem, and many studies dealing with this issue have been published with controversial outcomes. Therefore, more studies and new treatment strategies for stricture prevention are needed in the future.

Since the incidence of esophageal cancer is rising in the Western world, Western endoscopists have ambitiously strived to learn and improve their skills in EMR and ESD from their Japanese colleagues during the past few years and there are ongoing efforts to make these techniques broadly available in the West. Nevertheless, expanding the adoption of endoscopic tumor resection techniques in the West is challenging. This is mainly because of a limited availability of qualified Western mentors together with low rates of lesions that are suitable for removal by beginner EMR/ESD endoscopists. Moreover, as a future perspective, ESD training programs from official endoscopy societies are underway but not yet finally approved. These criteria are of tremendous importance for the adoption of EMR/ESD techniques to a broader field in the West. Importantly, also community endoscopists should be encouraged to improve their skills for early esophageal tumor detection, because they perform a substantial number of screening endoscopies.

Altogether, the development of EMR and ESD has essentially changed former therapy guidelines of early esophageal cancer and this has paved the way for minimally invasive, therapeutic oncologic tumor resection in this tumor entity. Therefore, endoscopists and endoscopy societies must continuously contribute toward spreading these skills to ensure optimal, nationwide tumor patient care in Western countries.

Conflict of interest T. Fritz, R. Schöfl, F. Wewalka, and A. Ziachehabi declare that they have no competing interests.

Open Access This article is distributed under the terms of the Creative Commons Attribution 4.0 International License (http://creativecommons.org/licenses/by/4.0/), which permits unrestricted use, distribution, and reproduction in any medium, provided you give appropriate credit to the original author(s) and the source, provide a link to the Creative Commons license, and indicate if changes were made.

\section{References}

1. Bailey SH, et al. Outcomes after esophagectomy: a ten-year prospective cohort. Ann Thorac Surg. 2003;75(1):217-22. discussion 222.

2. Rosenberg N. Submucosal saline wheal as safety factor in fulguration or rectal and sigmoidal polypi. AMA Arch Surg. 1955;70(1):120-2.

3. Yahagi N, Fujishiro M, Kakushima N, Kobayashi K, Hashimoto T, Oka M, Iguchi $\mathrm{M}$, Enomoto S, Ichinose M, Niwa H, Omata M. Endoscopic submucosal dissection for early gastric cancer using the tip of an electrosurgical snare (thin type). Dig Endosc. 2004;16:34-8.

4. Ono H, et al. Endoscopic mucosal resection for treatment of early gastric cancer. Gut. 2001;48(2):225-9.

5. Lian J, et al. A meta-analysis of endoscopic submucosal dissection and EMR for early gastric cancer. Gastrointest Endosc. 2012;76(4):763-70.

6. Park YM, et al. The effectiveness and safety of endoscopic submucosal dissection compared with endoscopic mucosal resection for early gastric cancer: a systematic review and metaanalysis. Surg Endosc. 2011;25(8):2666-77.

7. Ahlenstiel G, et al. Actual endoscopic versus predicted surgical mortality for treatment of advanced mucosal neoplasia of the colon. GastrointestEndosc. 2014;80(4):668-76.

8. Jayanna M, et al. Cost analysis of endoscopic mucosal resection vs surgery for large laterally spreading colorectal lesions. Clin GastroenterolHepatol. 2016;14(2):271-278e1-2.

9. Thomas T, et al. High-resolution endoscopy and endoscopic ultrasound for evaluation of early neoplasia in Barrett's esophagus. Surg Endosc. 2010;24(5):1110-6.

10. Puli SR, et al. Staging accuracy of esophageal cancer by endoscopic ultrasound: a meta-analysis and systematic review. World J Gastroenterol. 2008;14(10):1479-90.

11. Scotiniotis IA, etal. Accuracy ofEUSin the evaluation ofBarrett's esophagus and high-grade dysplasia or intramucosal carcinoma. GastrointestEndosc. 2001;54(6):689-96.

12. Peters FP, et al. Histologic evaluation of resection specimens obtained at 293 endoscopic resections in Barrett's esophagus. Gastrointest Endosc. 2008;67(4):604-9.

13. TheParis endoscopic classification of superficial neoplastic lesions: esophagus, stomach, and colon: November 30 to December 1, 2002. Gastrointest Endosc, 2003. 58(6): p. S3-S43.

14. Gono K, Yamazaki K, Doguchi N, Nonami T, Obi T, Yamaguchi M, Ohyama N, Machida H, Sano Y, Yoshida S, Hamamoto Y, Endo T. Endoscopic Observation of Tissue by Narrowband Illumination. OptRev. 2003;10(4):211-5.

15. Sato H, et al. Utility of intrapapillary capillary loops seen on magnifying narrow-band imaging in estimatinginvasive depth of esophageal squamous cell carcinoma. Endoscopy. 2015;47(2):122-8.

16. Mizumoto T, et al. Magnifying endoscopy with narrow band imaging in estimating the invasion depth of superficial esophageal squamous cell carcinomas. Digestion. 2018;98(4):249-56.

17. Endoscopic Classification Review G. Update on the paris classification of superficial neoplastic lesions in the digestive tract. Endoscopy. 2005;37(6):570-8.

18. Sharma P, et al. Development and validation of a classification system toidentifyhigh-grade dysplasiaand esophageal adenocarcinomain Barrett's esophagus using narrow-band imaging. Baillieres Clin Gastroenterol. 2016;150(3):591-8.

19. Kandiah $\mathrm{K}$, et al. International development and validation of a classification system for the identification of Barrett's neoplasia using acetic acid chromoendoscopy: 
the Portsmouth acetic acid classification (PREDICT). Gut. 2017; https://doi.org/10.1136/gutjnl-2017-314512.

20. Larghi A, et al. EUS followed by EMR for staging of highgrade dysplasia and early cancer in Barrett's esophagus. GastrointestEndosc. 2005;62(1):16-23.

21. Dubecz A, et al. Does the incidence of adenocarcinoma of the esophagus and gastric cardia continue to rise in the twenty-first century? - a SEER database analysis. J Gastrointest Surg. 2013;18:124. https://doi.org/10.1007/ s11605-013-2345-8.

22. Desai TK, et al. The incidence of oesophageal adenocarcinoma in non-dysplastic Barrett's oesophagus: a metaanalysis. Gut. 2012;61(7):970-6.

23. Weusten B, et al. Endoscopic management of Barrett's esophagus: European Society of Gastrointestinal Endoscopy (ESGE) Position Statement. Endoscopy. 2017;49(2):191-8.

24. Bhat SK, et al. Oesophageal adenocarcinoma and prior diagnosis of Barrett's oesophagus: a population-based study. Gut. 2015;64(1):20-5.

25. Dulai GS, et al. Preoperative prevalence of Barrett's esophagus in esophageal adenocarcinoma: a systematic review. Baillieres Clin Gastroenterol. 2002;122(1):26-33.

26. Pimentel-Nunes P, et al. Endoscopic submucosal dissection: European Society of Gastrointestinal Endoscopy (ESGE) Guideline. Endoscopy. 2015;47(9):829-54.

27. Duits LC, etal. Barrett's oesophagus patients withlow-grade dysplasia can be accurately risk-stratified after histological reviewby an expert pathology panel. Gut. 2015;64(5):700-6.

28. Inoue H. Endoscopic mucosal resection for the entire gastrointestinal mucosal lesions. Gastrointest Endosc Clin NAm. 2001;11(3):459-78.

29. Seewald S, et al. Circumferential EMR and complete removal of Barrett's epithelium: a new approach to management of Barrett's esophagus containing high-grade intraepithelial neoplasia and intramucosal carcinoma. GastrointestEndosc. 2003;57(7):854-9.

30. Abrams JA, et al. Depth of resection using two different endoscopic mucosal resection techniques. Endoscopy. 2008;40(5):395-9.

31. May A, et al. A prospective randomized trial of two different endoscopic resection techniques for early stage cancer of the esophagus. Gastrointest Endosc. 2003;58(2):167-75.

32. Pouw RE, et al. Randomized trial on endoscopic resectioncap versus multiband mucosectomy for piecemeal endoscopic resection of early Barrett's neoplasia. Gastrointest Endosc. 2011;74(1):35-43.

33. Pech $\mathrm{O}$, et al. Long-term efficacy and safety of endoscopic resection for patients with mucosal adenocarcinoma of the esophagus. Baillieres Clin Gastroenterol. 2014;146(3):652-660e1.

34. Phoa KN, et al. Radiofrequency ablation vs endoscopic surveillance for patients with Barrett esophagus and lowgrade dysplasia: a randomized clinical trial. JAMA. 2014;311(12):1209-17.

35. Shaheen NJ, et al. Radiofrequency ablation in Barrett's esophagus with dysplasia. NEnglJMed. 2009;360(22):2277-88.

36. Small AJ, et al. Radiofrequency ablation is associated with decreased neoplastic progression in patients with Barrett's esophagus and confirmed low-grade dysplasia. Baillieres Clin Gastroenterol. 2015;149(3):567-576e3. quize13-4.

37. Shaheen NJ, et al. Durability of radiofrequency ablation in Barrett's esophagus with dysplasia. Baillieres Clin Gastroenterol. 2011;141(2):460-8.

38. Pech $\mathrm{O}$, et al. Long-term results and risk factor analysis for recurrence after curative endoscopic therapy in 349 patients with high-grade intraepithelial neoplasia and mucosal adenocarcinoma in Barrett's oesophagus. Gut. 2008;57(9):1200-6.

39. Griffin SM, Burt AD, Jennings NA. Lymph node metastasis in early esophageal adenocarcinoma. Ann Surg. 2011;254(5):731-6. discussion736-7.

40. Zemler B, et al. Early Barrett's carcinoma: the depth of infiltration of the tumour correlates with the degree of differentiation, the incidence of lymphatic vessel and venous invasion. Virchows Arch. 2010;456(6):609-14.

41. Gamboa AM, et al. Treatment allocation in patients with early-stage esophageal adenocarcinoma: prevalence and predictors of lymph node involvement. Cancer. 2016;122(14):2150-7.

42. Manner H, et al. Efficacy, safety, and long-term results of endoscopic treatment for early stage adenocarcinoma of the esophagus with low-risk sm1 invasion. Clin Gastroenterol Hepatol. 2013;11(6):630-5. quize45.

43. Terheggen G, et al. A randomised trial of endoscopic submucosal dissection versus endoscopic mucosal resection for early Barrett's neoplasia. Gut. 2017;66(5):783-93.

44. Cao Y, et al. Meta-analysis of endoscopic submucosal dissection versus endoscopic mucosal resection for tumors of the gastrointestinal tract. Endoscopy. 2009;41(9):751-7.

45. Morita FH, et al. Narrow band imaging versus lugol chromoendoscopy to diagnose squamous cell carcinoma of the esophagus: a systematic review and meta-analysis. BMCCancer. 2017;17(1):54.

46. Inoue $\mathrm{H}$, et al. Magnification endoscopy in esophageal squamous cell carcinoma: a review of the intrapapillary capillary loop classification. Ann Gastroenterol. 2015;28(1):41-8.

47. Takahashi H, et al. Endoscopic submucosal dissection is superior to conventional endoscopic resection as a curative treatment for early squamous cell carcinoma of the esophagus (with video). Gastrointest Endosc. 2010;72(2):255-264, 264e1-2.

48. TajimaY, etal. Significance ofinvolvementbysquamouscell carcinoma of the ducts of esophageal submucosal glands. Analysis of 201 surgically resected superficial squamous cell carcinomas. Cancer. 2000;89(2):248-54.

49. Natsugoe S, et al. Mucosal squamous cell carcinoma of the esophagus: a clinicopathologic study of 30 cases. Oncology. 1998;55(3):235-41.

50. Bollschweiler E, et al. High rate of lymph-node metastasis in submucosal esophageal squamous-cell carcinomas and adenocarcinomas. Endoscopy. 2006;38(2):149-56.

51. Yang D, et al. Endoscopic submucosal dissection for early Barrett's neoplasia: a meta-analysis. Gastrointest Endosc. 2018;87(6):1383-93.

52. Ono S, et al. Predictors of postoperative stricture after esophageal endoscopic submucosal dissection for superficial squamous cell neoplasms. Endoscopy. 2009;41(8):661-5.

53. Yamaguchi N, et al. Usefulness of oral prednisolone in the treatment of esophageal stricture after endoscopic submucosal dissection for superficial esophageal squamous cell carcinoma. GastrointestEndosc. 2011;73(6):1115-21.

54 . Hashimoto S, et al. The efficacy of endoscopic triamcinolone injection for the prevention of esophageal stricture after endoscopic submucosal dissection. Gastrointest Endosc. 2011;74(6):1389-93.

55. Deprez PH. Esophageal strictures after extensive endoscopic resection: hope for a better outcome? Gastrointest Endosc. 2013;78(2):258-9.

56. Kuribayashi Y, et al. Esophageal motility after extensive circumferential endoscopic submucosal dissection for superficial esophageal cancer. Digestion. 2018;98(3):153-60. 